\title{
ARTICLE Icariside II attenuates cerebral ischemia/reperfusion-induced blood-brain barrier dysfunction in rats via regulating the balance of MMP9/TIMP1
}

Mu-bo Liu ${ }^{1,2}$, Wei Wang ${ }^{2,3}$, Jian-mei Gao ${ }^{1,2,3}$, Fei $\mathrm{Li}^{2,3}$, Jing-shan Shi ${ }^{2,3}$ and Qi-hai Gong ${ }^{1,2,3}$

\begin{abstract}
Cerebral ischemia/reperfusion (I/R) results in harmful consequences during ischemic stroke, especially the disruption of the blood-brain barrier (BBB), which leads to severe hemorrhagic transformation through aggravation of edema and brain hemorrhage. Our previous study demonstrated that icariside II (ICS II), which is derived from Herba Epimedii, attenuates cerebral I/R injury by inhibiting the GSK-3 $\beta$-mediated activation of autophagy both in vitro and in vivo. However, the effect of ICS II on the BBB remains unclear. Thus, in this study, we investigated the regulation of BBB integrity by ICS II after cerebral I/R injury and further explored the underlying mechanism in rats. Cerebral I/R injury was induced by middle cerebral artery occlusion (MCAO), and the treatment groups were administered ICS II at a dose of $16 \mathrm{mg} / \mathrm{kg}$ by gavage twice a day for 3 days. The results showed that ICS II effectively prevented BBB disruption, as evidenced by Evans Blue staining. Moreover, ICS II not only significantly reduced the expression of MMP2/9 but also increased TIMP1 and tight junction protein (occludin, claudin 5, and ZO 1) expression. Intriguingly, ICS II may directly bind to both MMP2 and MMP9, as evidenced by molecular docking. In addition, ICS II also inhibited cerebral I/R-induced apoptosis and ameliorated the Bax/Bcl-2 ratio and cleaved-caspase 3 level. Collectively, our findings reveal that ICS II significantly ameliorates I/R-induced BBB disruption and neuronal apoptosis in MCAO rats by regulating the MMP9/TIMP1 balance and inhibiting the caspase 3-dependent apoptosis pathway.
\end{abstract}

Keywords: Stroke; cerebral ischemia/reperfusion; MCAO rats; icariside Il; blood-brain barrier; apoptosis; tight junction; molecular docking

Acta Pharmacologica Sinica (2020) 41:1547-1556; https://doi.org/10.1038/s41401-020-0409-3

\section{INTRODUCTION}

Stroke, the second leading cause of death and a frequent cause of permanent disability in China, results from the rupture or occlusion of cerebral vessels and can be categorized as ischemic stroke or hemorrhagic stroke depending on its pathological features [1, 2]. Ischemic strokes account for $\sim 85 \%$ of strokes, and it has long been recognized that ischemic stroke results in severe outcomes even when thrombolysis is used; it has a mortality rate of up to a $25 \%$, and $\sim 50 \%$ of patients are left with permanent disability [3]. Unfortunately, there are currently no ideal medical treatments available for ischemic stroke, and intravenous thrombolysis with recombinant tissue plasminogen activator (tPA) is the only FDAapproved pharmacotherapy for ischemic stroke $[4,5]$. Despite these promising findings, tPA therapy has a narrow time window and is associated with severe adverse reactions, such as hemorrhagic transformation and cerebral ischemia/reperfusion (I/R) injury, and tPA restores blood flow to the ischemic tissue, inducing additional serious injury to the brain [6-9]. Thus, it is necessary to exploit new therapeutic approaches or drugs for ischemic stroke.

Emerging evidence shows that the blood-brain barrier (BBB), a dynamic and complex interface, is a unique and selective barrier between the blood and the brain that regulates the nutrients and toxins in and out of the central nervous system (CNS) [10-12]. Importantly, the BBB plays a crucial role in maintaining stable conditions in the CNS [13]. Under pathological conditions, such as stroke, the BBB can be disrupted, which facilitates injury progression, increases brain infarction and edema, and increases the risk of hemorrhagic transformation and even death [14-16]. From this perspective, the attenuation of BBB dysfunction may be a potential therapeutic target for ischemic stroke.

Icariside II (ICS II), a flavonoid derived from Herba Epimedii, has many beneficial activities, such as anti-osteoporotic, anti-aging, and anti-inflammatory properties and effects against erectile dysfunction [17-19]. Recently, ICS II has drawn increasing attention due to its remarkable beneficial effects in neurodegenerative diseases such as Alzheimer's disease [20,21]. Of note, our preliminary investigations suggested that ICS II can attenuate cerebral $\mathrm{l} / \mathrm{R}$ injury by inhibiting the glycogen synthase kinase (GSK)-3 $\beta$-mediated activation of autophagy both in vitro and in vivo [22]. However, whether ICS II can alleviate BBB dysfunction during cerebral I/R injury remains unclear. Therefore, the present study aimed to investigate the effect of ICS II on cerebral ischemia

\footnotetext{
${ }^{1}$ Department of Pharmacology, School of Pharmacy, Zunyi Medical University, Zunyi 563000, China; ${ }^{2}$ Key Laboratory of Basic Pharmacology of Ministry of Education and Joint International Research Laboratory of Ethnomedicine of Ministry of Education, Zunyi Medical University, Zunyi 563000, China and ${ }^{3}$ Key Laboratory of Basic Pharmacology of Guizhou Province, Zunyi Medical University, Zunyi 563000, China

Correspondence: Qi-hai Gong (gqh@zmu.edu.cn)
}

Received: 18 December 2019 Accepted: 26 March 2020

Published online: 2 June 2020 
a

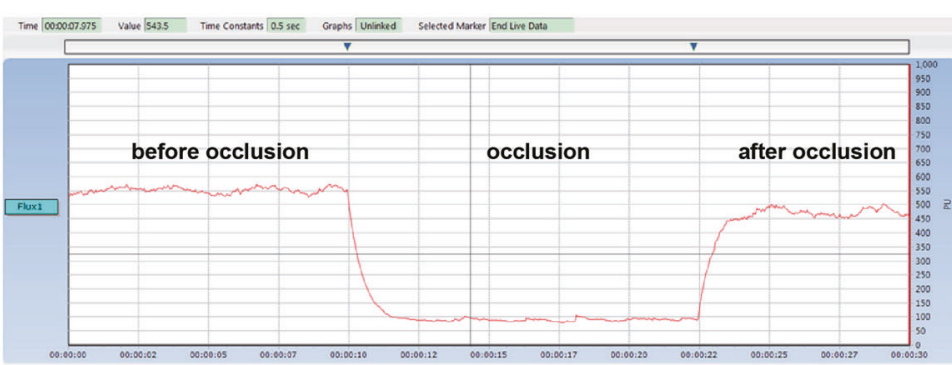

C

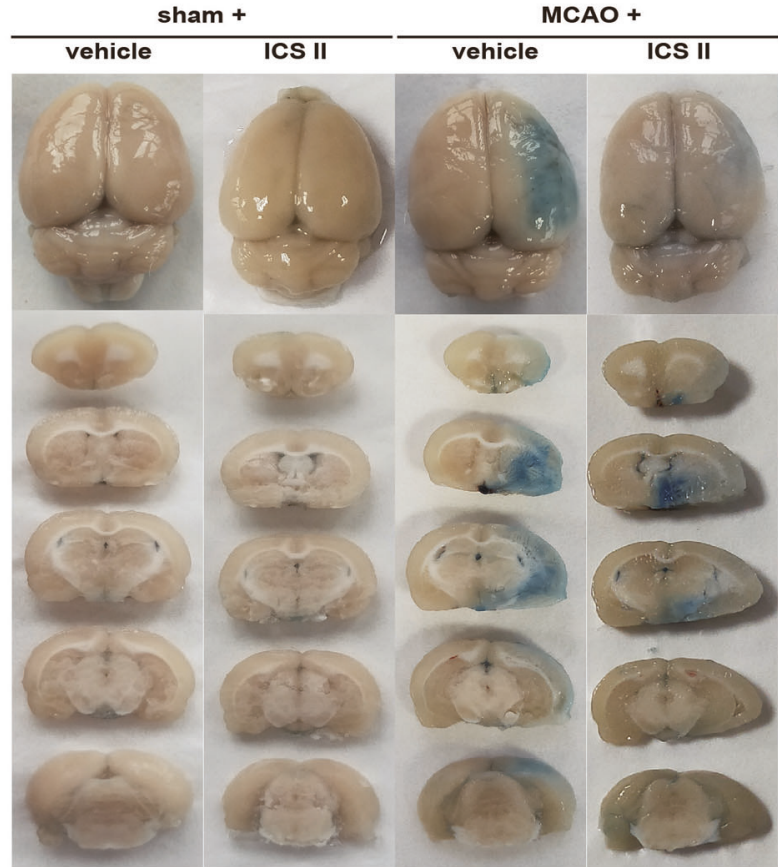

b

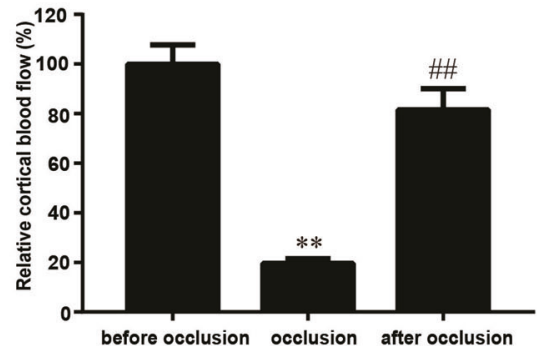

d

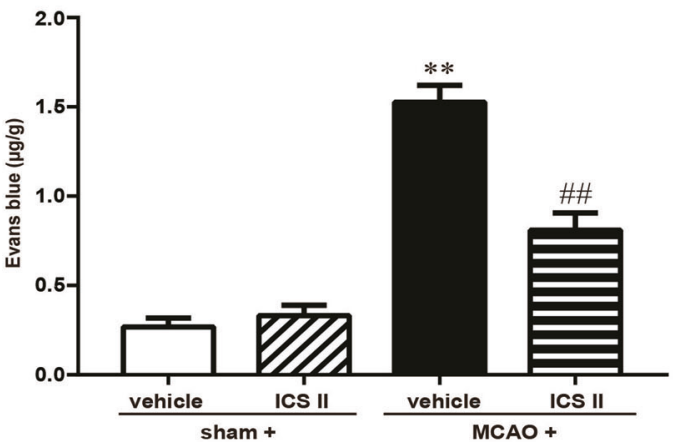

Fig. 1 Cortical blood flow before and after MCAO and the protective effect of ICS II against BBB damage after ischemic brain injury induced by cerebral I/R in rats. a Representative images of cortical blood flow. $\mathbf{b}$ Quantitative analysis of relative cortical blood flow (\%). ${ }^{* *} P<$ 0.01 vs before occlusion, ${ }^{\# \#} P<0.01$ vs occlusion, $n=5$. c Representative images of Evans blue extravasation in the rat brain. $\mathbf{d}$ Quantitative analysis of Evans blue dye extravasation into the brain hemisphere. ${ }^{* *} P<0.01$ vs sham; ${ }^{\# \#} P<0.01$ vs MCAO; $n=6$ per group.

I/R-induced BBB dysfunction in rats and to further explore its underlying mechanism.

\section{MATERIALS AND METHODS}

Animals and drug

Male Sprague-Dawley rats (250-280 g) were provided by Changsha Tianqin Biotechnology Co., Ltd (Changsha, China; Certificate No. SCXK2014-0011). Before experimentation, the rats were allowed to acclimatize to the experimental conditions (temperature of $25 \pm 1{ }^{\circ} \mathrm{C}$, relative humidity of $55 \% \pm 5 \%$, and a 12 -h lightdark cycle in an animal room) for a week. Laboratory chow and tap water were provided ad libitum. All of the animal experiments were approved by the Institutional Animal Ethics and Use Committee of Zunyi Medical University. ICS II (purity $\geq 98 \%$ by HPLC) was purchased from Nanjing Manhay Medical Technology Co., Ltd (Nanjing, China) and dissolved in normal saline using ultrasonication for $20 \mathrm{~min}$. Monofilament nylon sutures were obtained from Beijing Cinontech Co., Ltd (Beijing, China). MMP2 (ab37150), MMP9 (ab38898), TIMP1 (ab61224), caspase 3 (ab13847), cleaved-caspase 3 (ab2302), Bcl-2 (ab59348), Bax (ab53154), and occludin (ab167161) antibodies were obtained from Abcam Inc. (Cambridge, USA). A claudin 5 antibody (AF5216) was obtained from Affinity Biosciences (Cincinnati, OH, USA).
Evans blue dye was obtained from Dalian Meilun Biotechnology. Co., Ltd (Dalian, China). Bicinchoninic acid (BCA) protein concentration analysis kits, ZO 1 (21773-1-AP) and a secondary antibody were purchased from Proteintech Group, Inc (Rosemont, IL, USA).

Rat MCAO model

In this study, an experimental model of cerebral I/R injury was induced in rats by middle cerebral artery occlusion (MCAO). The surgical procedure was performed according to our previous study [23]. In brief, after intraperitoneal anaesthetization with 7\% choral hydrate, the right external carotid artery (ECA) and internal carotid artery (ICA) of each rat were exposed. A monofilament nylon suture (diameter of $0.26 \mathrm{~mm}$ ) with a spherical tip (diameter of $0.36 \pm 0.02 \mathrm{~mm}$ ) was inserted into the ECA to block the flow of blood to the middle cerebral artery. The monofilament was pulled out after $2 \mathrm{~h}$ of occlusion, and this was followed by reperfusion for 3 days. The rats in the sham group underwent the same surgical procedure except for insertion of the monofilament. To monitor cortical blood flow, a laser-doppler flowmeter (Moor Instruments, Ltd., Millwey, Axminster, UK) was used.

ICS II administration and groups

The rats were subjected to the surgical procedure and divided into four groups: the sham, model (vehicle), sham + ICS II, and model + 
a

sham +

$\mathrm{MCAO}+$

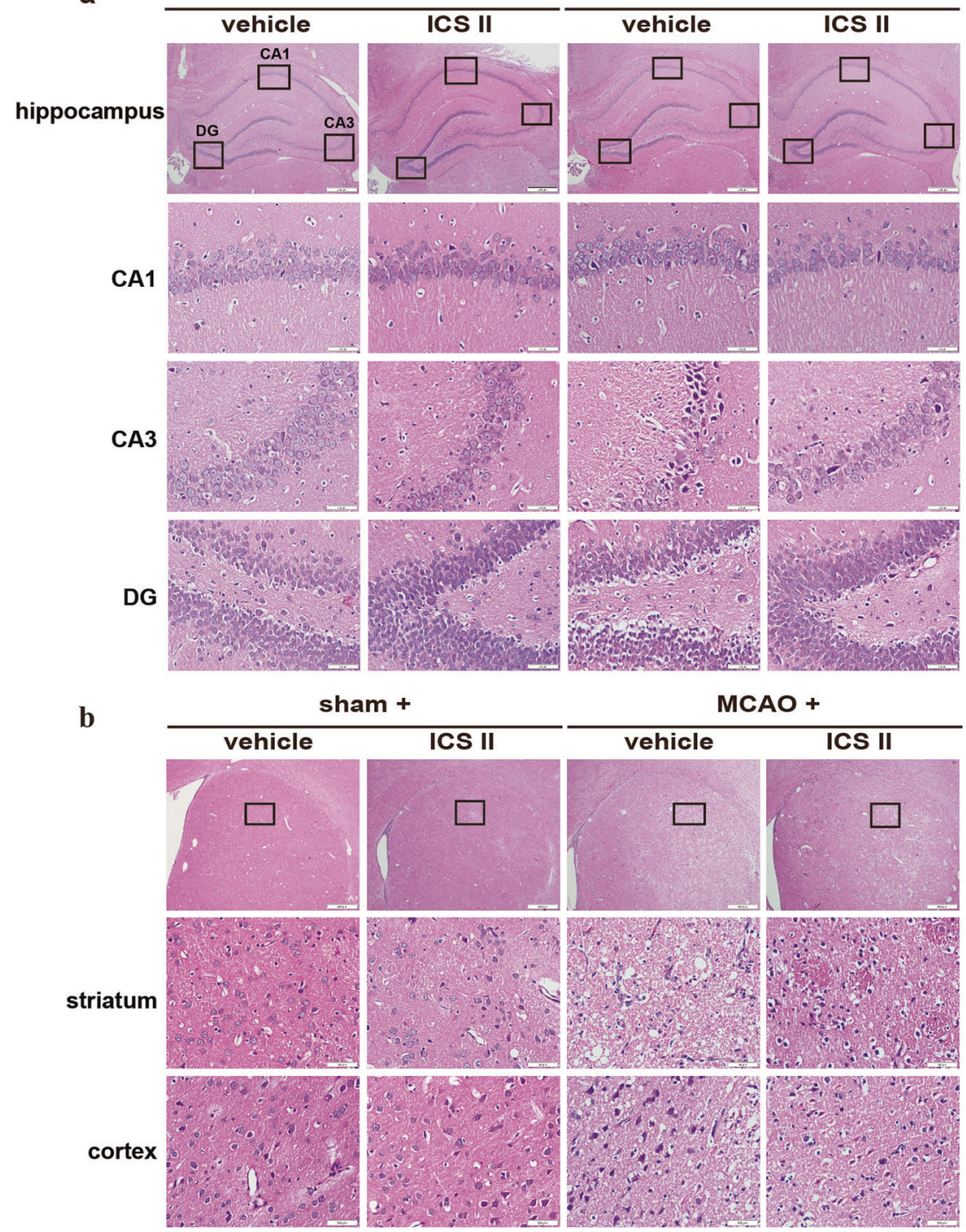

Fig. 2 ICS II suppressed damage to the hippocampus, cortex and striatum after cerebral I/R injury. a The hippocampal CA1, CA3 and DG regions. b Striatum and cortex $(\times 400$, scale bar $=50 \mu \mathrm{m}) . n=6$ per group.

ICS II groups. The sham + ICS II and model + ICS II groups were treated with ICS II at a dose of $16 \mathrm{mg} / \mathrm{kg}$ by gavage twice a day for 3 days, while the animals in the sham and model groups received the same volume of normal saline.

\section{Assessment of BBB integrity}

BBB disruption was quantitatively determined by Evans blue staining as described previously. Briefly, Evans blue dye $(2 \%, 4 \mathrm{~mL} /$ $\mathrm{kg}$ ) was injected through the tail vein. After $2 \mathrm{~h}$ of circulation, the rats were anaesthetized and perfused with PBS solution. The brains were rapidly isolated, imaged and then weighed and homogenized in formamide. After centrifugation, the supernatant was collected and measured with an enzyme-labeled instrument (Multiskan GO, Thermo, USA) at $620 \mathrm{~nm}$.

Histological examination and Nissl staining

In brief, the rat brains were immediately perfused with PBS followed by $4 \%$ paraformaldehyde buffer at $4{ }^{\circ} \mathrm{C}$ for $48 \mathrm{~h}$, dehydrated and embedded in paraffin. Subsequently, 3.5- $\mu \mathrm{m}$ thick sections were prepared and treated with xylene and ethanol.
These slices were stained with hematoxylin and eosin (H\&E) at room temperature and then stained with $1 \%$ toluidine blue at 60 ${ }^{\circ} \mathrm{C}$ for $15 \mathrm{~min}$. Histopathology was observed using a light microscope.

Immunohistochemical staining

Likewise, the brains were perfused with PBS followed by $4 \%$ paraformaldehyde, dehydrated and embedded in paraffin. Subsequently, 3.5- $\mu \mathrm{m}$ thick sections were prepared and treated with xylene and ethanol. Then, the slices were washed with double distilled water, incubated with $3 \% \mathrm{H}_{2} \mathrm{O}_{2}$ for $17 \mathrm{~min}$, and subjected to antigen repair with citrate buffer in a microwave for $8 \mathrm{~min}$ three times. Thereafter, the slices were blocked with goat serum for 30 min and then incubated with primary antibodies against MMP2 (1:200), MMP9 (1:500) and TIMP1 (1:300) at $4{ }^{\circ} \mathrm{C}$ for $17 \mathrm{~h}$. Then, the sections were incubated with secondary goat anti-rabbit lgG and HRP-labeled streptozotocin for $20 \mathrm{~min}$ at $37^{\circ} \mathrm{C}$ after being washed with PBS three times and then visualized using a DAB kit. Finally, images were analyzed by light microscopy and Image Pro Plus software for statistical analysis. 

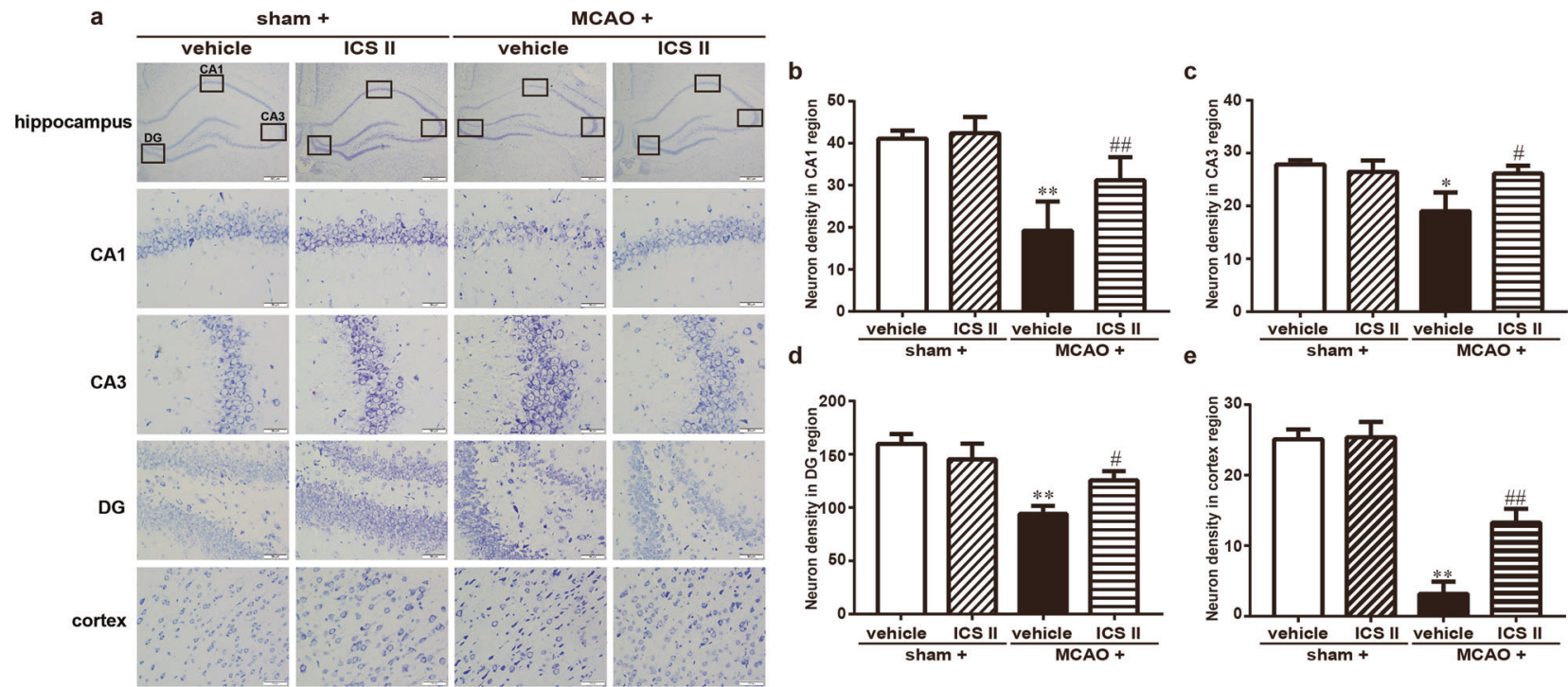

Fig. 3 ICS II protected against cerebral I/R injury-induced neuronal loss in the hippocampus and cortex. a The hippocampal CA1, CA3, and $D G$ regions and cortex. $\mathbf{b}$ Quantitative analysis of neuronal density in the CA1 region. $\mathbf{c}$ Quantitative analysis of neuronal density in the CA3 region. d Quantitative analysis of neuronal density in the DG region. e Quantitative analysis of neuronal density in the cortex. ${ }^{*} P<0.05$, ${ }^{*} P<$ 0.01 vs sham; ${ }^{\#} P<0.05,{ }^{\# \#} P<0.01$ vs $M C A O ; n=6$ per group.

Western blot analysis

Protein samples were extracted from the ischemic penumbra using RIPA buffer supplemented with the proteinase inhibitor PMSF. After incubation and centrifugation, the supernatant was collected, and a BCA assay kit was used to quantify the total protein levels. Equal quantities of total protein $(30 \mu \mathrm{g})$ were separated on $6 \%-12 \%$ SDSpolyacrylamide gels and incubated with primary antibodies against MMP2 (1:1000), MMP9 (1:1000), TIMP1 (1:2000), claudin 5 (1:2000), occludin (1:50,000), ZO 1 (1:1000), caspase 3 (1:1000), cleavedcaspase 3 (1:2000), Bcl-2 (1:1000), and Bax (1:1000) followed by a secondary antibody (HRP-labeled goat anti-rabbit lgG, 1:5000). The immunoreactive bands were visualized with $\mathrm{ECL}$ detection reagents and quantified on a ChemiDoc MP Imaging System (Bio-Rad Laboratories, Inc., Hercules, CA, USA).

TUNEL staining

Cellular apoptosis was evaluated by an In Situ Cell Death Detection Kit, POD (Roche Diagnostics, Germany). In brief, paraffin-embedded tissue slices were treated with xylene and ethanol. Then, the cells were incubated with $3 \% \mathrm{H}_{2} \mathrm{O}_{2}$ for 20 min and proteinase $\mathrm{K}$ for $13 \mathrm{~min}$ at $37^{\circ} \mathrm{C}$. The TUNEL reaction mixture and Converter-POD were added, and slices were visualized with a DAB kit. Finally, the samples were analyzed by light microscopy.

\section{Molecular docking}

Molecular docking calculations were performed using Autodock 4.2, and the affinity between ICS II and MMP2/9 was observed using AutodockTools software. The three-dimensional (3D) protein structures of MMP2 (PDB ID: 2ZHV) and MMP9 (PDB ID: 1L6J) were retrieved from the Protein Data Bank (https://www.rcsb.org). The molecular docking of the ICS II and MMP2/9 binding sites was analyzed using Autodock software.

Statistical analysis

All values are presented as the mean \pm standard error of the mean (SEM) and were analyzed using SPSS version 18.0 (SPSS, Inc., Chicago, USA). The data were analyzed by one-way analysis of variance, and differences among means were analyzed using the LSD (equal variance assumed) and the Dunnett T3 test (equal variance not assumed). $P<0.05$ was considered a statistically significant difference.

\section{RESULTS}

ICS II attenuated BBB disruption after cerebral I/R injury in rats The rats were subjected to cerebral $I / R$ injury as described in a previous study [23]. Thereafter, BBB disruption was detected by Evans blue staining, and the results showed that after MCAO, the ischemic brain hemisphere displayed much more severe leakage of Evans Blue dye than in the sham group, indicating that cerebral I/R disrupted the BBB. However, ICS II obviously prevented the cerebral I/R injury-induced increase in Evans Blue extravasation (Fig. 1). These findings suggested that ICS II protected against cerebral I/R injury in rats, at least partly, through mitigating BBB damage.

ICS II suppressed damage to the hippocampus, cortex and striatum after cerebral I/R injury

Cerebral I/R injury-induced neuropathological changes were assessed by $H \& E$ staining. The results showed that neurons in the hippocampus, cortex and striatum exhibited clear boundaries and normal morphology, while most of the neurons in the hippocampus, cortex and striatum disappeared or lacked a visible cell boundary after MCAO insult. Notably, ICS II reversed these changes in the hippocampus (Fig. 2a), striatum and cortex (Fig. 2b), which suggested that ICS II significantly suppressed damage to the hippocampus, cortex and striatum after cerebral I/R injury.

ICS II inhibited cerebral I/R-induced neuronal loss in the hippocampus and cortex

Cerebral l/R-induced neuronal loss was assessed by Nissl staining. The results showed a loss of Nissl bodies, neuronal atrophy and shrunken nuclei in the hippocampus and cortex after MCAO. However, ICS II markedly enhanced the number of normal NissI bodies compared with that in the MCAO group (Fig. 3).

ICS II attenuated the MMP/TIMP1 balance in cerebral I/R-induced injury

The expression and localization of MMP2/9 and TIMP1 were detected by Western blotting and immunohistochemistry, respectively. The results suggested that active MMP2/9 were enhanced and TIMP1 was diminished after MCAO compared with after sham surgery, while the active MMP/TIMP1 ratio after MCAO insult was significantly attenuated by ICS II (Fig. 4). Moreover, the expression of MMP2 and MMP9 in the cortex and striatum was significantly 
a

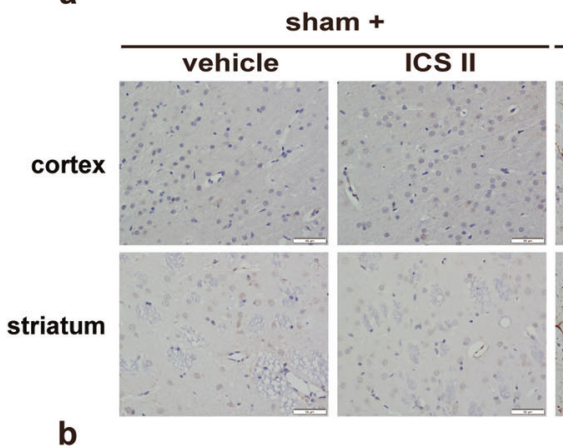

b

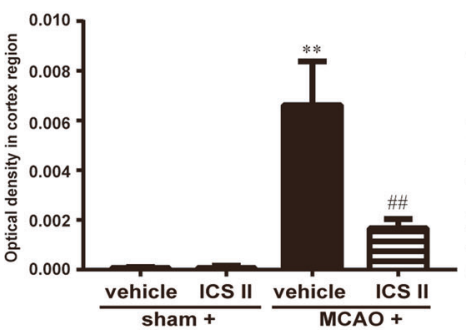

e

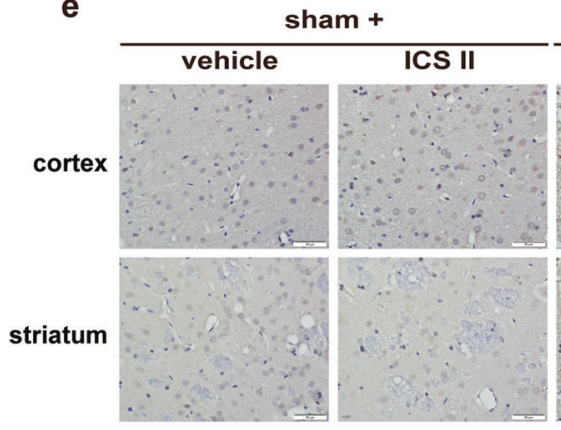

g

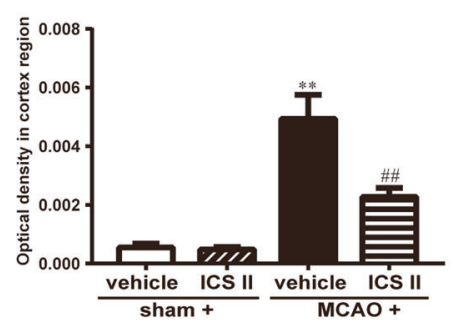

$\mathrm{MCAO}+$
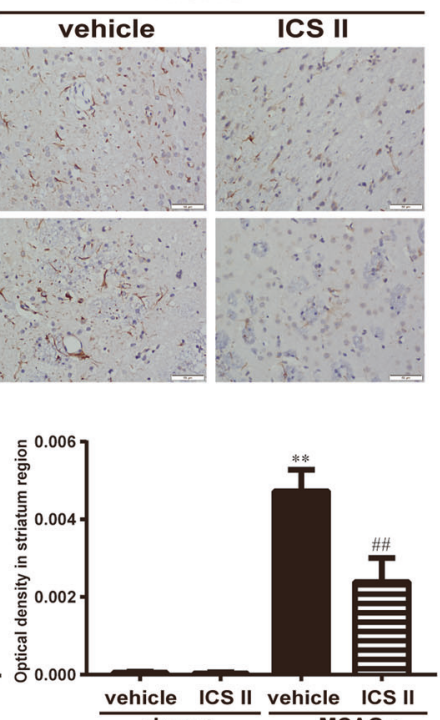

sham + $\frac{\text { MCAO + }}{\text { MCA }}$
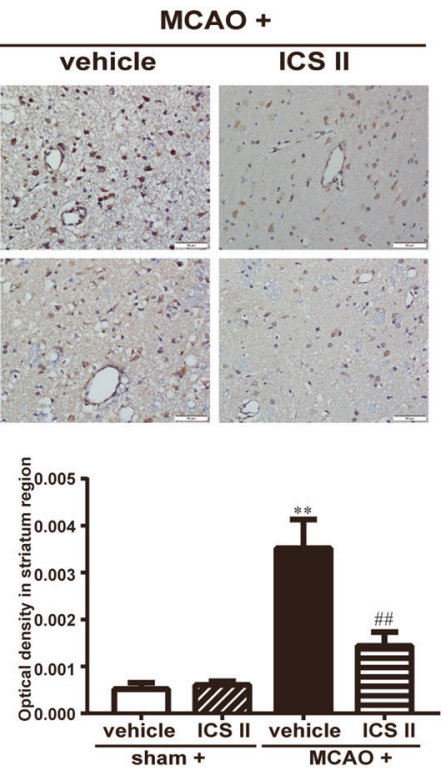

C

Fig. 4 ICS II attenuated MMP2/9 expression in cerebral I/R-induced injury. a Representative images of immunohistochemical staining of MMP9 in the cortex and striatum. b Quantitative analysis of MMP9 in the cortex and striatum. $\mathbf{c}$ Representative images of immunoblotting of MMP9 in the penumbra. d Quantitative analysis of MMP9 in the penumbra. e Representative images of immunohistochemical staining of MMP2 in the cortex and striatum. f Quantitative analysis of MMP2 in the cortex and striatum. $\mathbf{g}$ Representative images of immunoblotting of MMP2 in the penumbra. $\mathbf{h}$ Quantitative analysis of MMP2 in the penumbra. Five random microscope fields per section were chosen. The average was calculated. ${ }^{* *} P<0.01$ vs sham; ${ }^{\#} P<0.05$, ${ }^{\# \#} P<0.01$ vs MCAO; $n=6$ per group.

increased and TIMP1 expression was decreased after MCAO, as evidenced by immunohistochemical staining, whereas ICS II remarkably decreased the expression of MMP2/9 and increased the expression of TIMP1 in the cortex and striatum, respectively (Fig. 5). These results indicated that ICS II mediated the MMP/ TIMP1 balance during cerebral I/R injury, particularly in the cortex and striatum.

ICS II improved BBB integrity after cerebral I/R by upregulating the expression of tight junction-related proteins

The results suggested that the expression of tight junction proteins, including occludin, claudin 5 and ZO 1, was decreased after MCAO insult, as evidenced by Western blot analysis. However, ICS II markedly increased the expression of occludin, claudin 5 and ZO 1 (Fig. 6), which indicated that ICS II improved BBB integrity after cerebral I/R.

ICS II inhibited cerebral I/R-induced neural apoptosis in rats The results demonstrated that the number of apoptotic cells was remarkably significantly increased after MCAO compared with that in the sham group, as evidenced by the TUNEL assay (Fig. 7a). However, ICS II obviously reduced the number of apoptotic cells compared with that in the MCAO group. Furthermore, ICS II not only increased the ratio of $\mathrm{Bax} / \mathrm{Bcl}-2$ but also decreased the level of active caspase 3 compared with that in the MCAO group, as evidenced by Western blot analysis (Fig. 7c, d). These findings suggested that ICS II significantly suppressed cerebral I/R-induced neural apoptosis. 


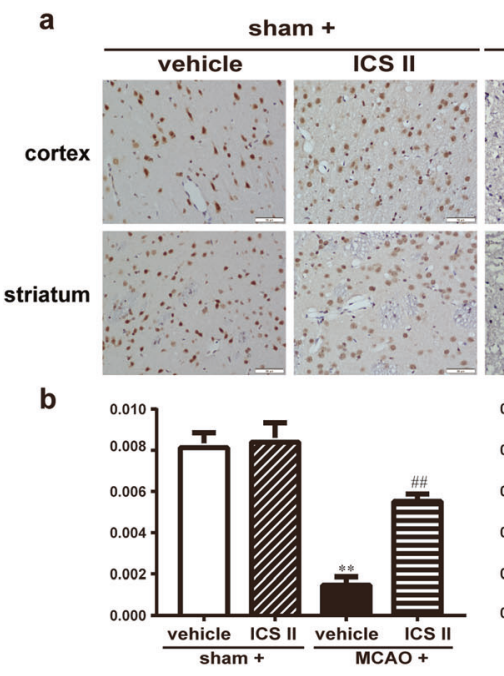

e

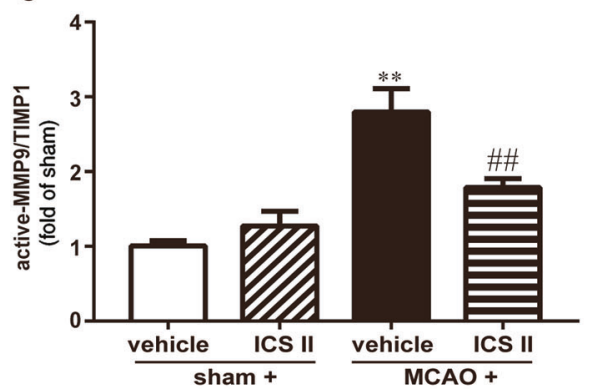

C

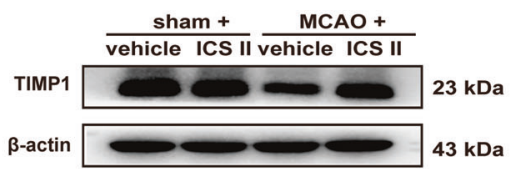

d

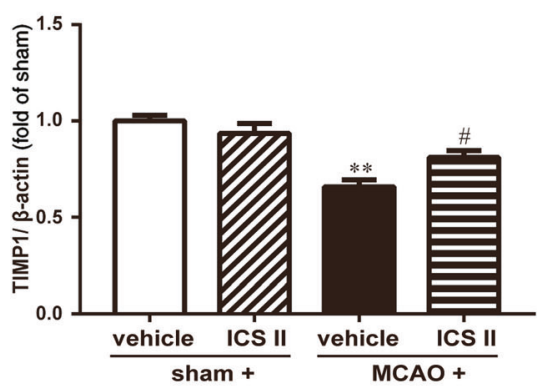

f

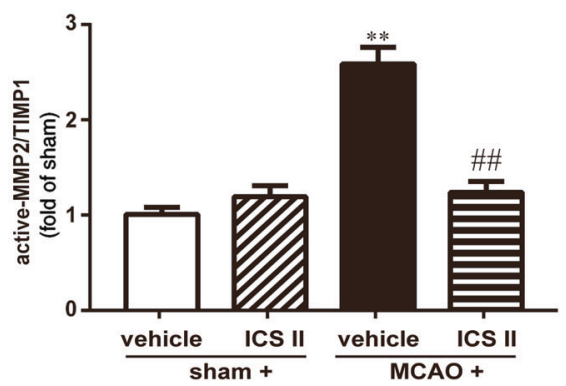

Fig. 5 ICS II increased TIMP1 expression in cerebral I/R-induced injury. a Representative images of immunohistochemical staining of TIMP1 in the cortex and striatum. b Quantitative analysis of TIMP1 in the cortex and striatum. c Representative images of immunoblotting of TIMP1 in the penumbra. d Quantitative analysis of TIMP1 in the penumbra. e Quantitative analysis of active MMP9/TIMP1 in the penumbra. f Quantitative analysis of active MMP2/TIMP1 in the penumbra. Five random microscope fields per section were chosen. The average was calculated. ${ }^{* *} P<0.01$ vs sham; ${ }^{\#}<0.05,{ }^{\# \#} P<0.01$ vs MCAO; $n=6$ per group.
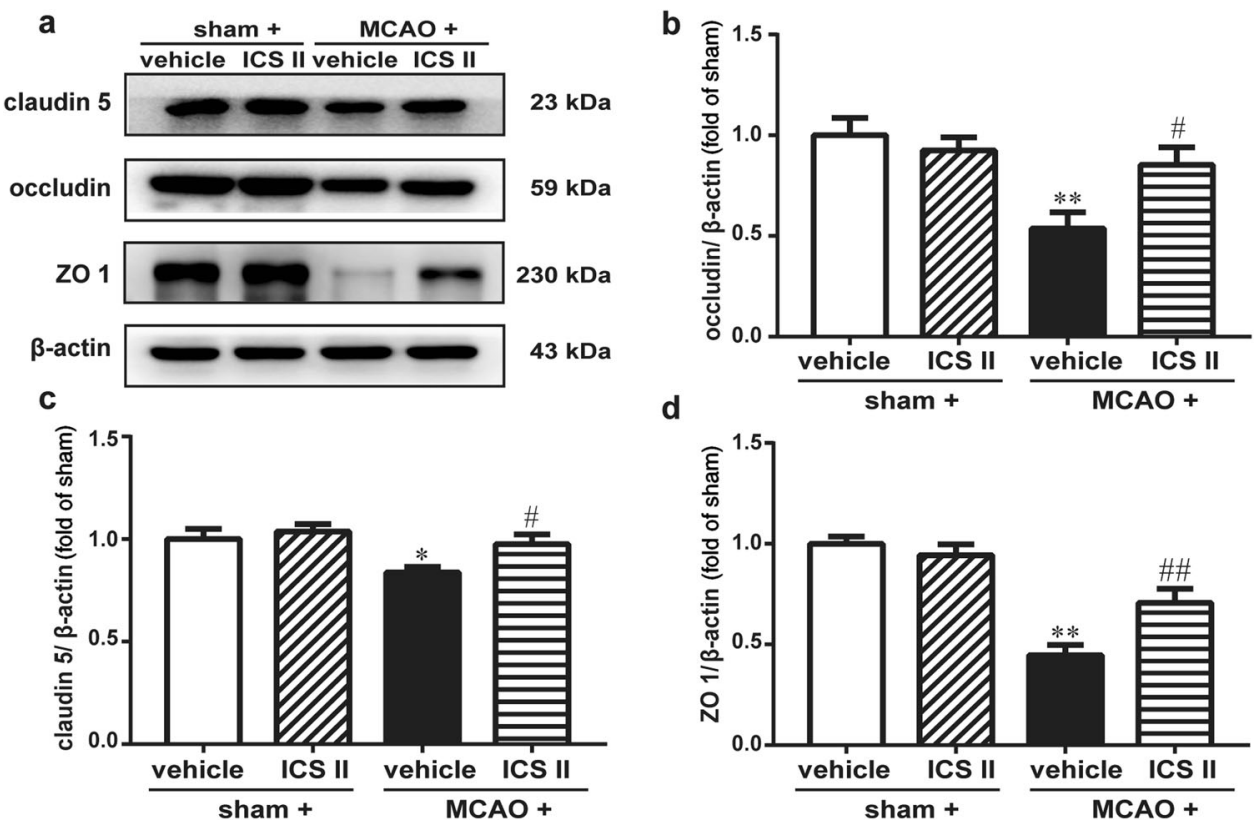

d

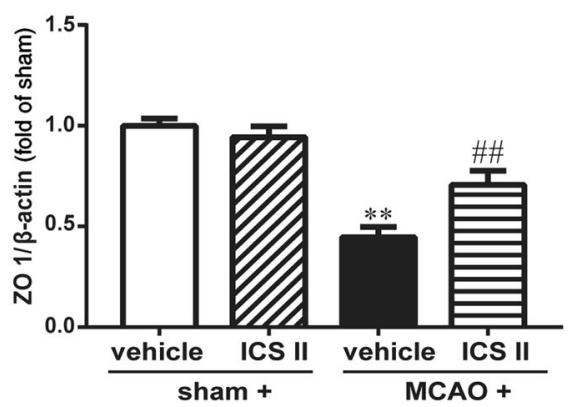

Fig. 6 ICS II improved BBB integrity after cerebral I/R by upregulating the expression of tight junction-related proteins. a Representative images of immunoblotting staining of claudin 5, occludin and $\mathrm{ZO} 1$ in the penumbra. b Quantitative analysis of occludin. c Quantitative analysis of claudin 5. d Quantitative analysis of ZO 1 . ${ }^{*} P<0.05,{ }^{* *} P<0.01$ vs sham; ${ }^{\#} P<0.05,{ }^{\# \#} P<0.01$ vs MCAO; $n=6$ per group. 


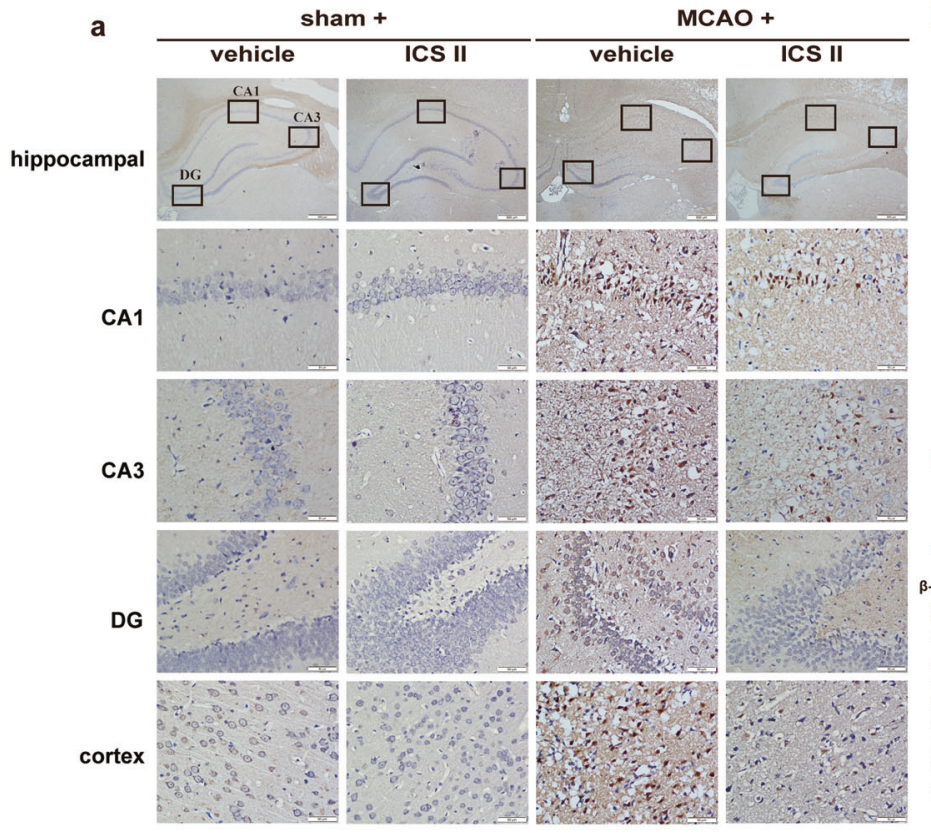

b
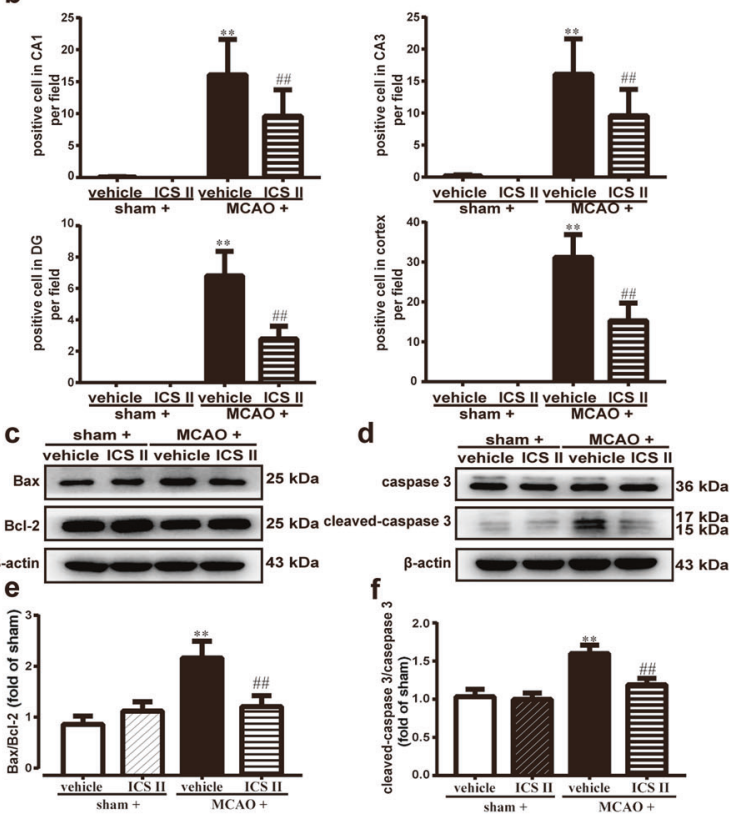

Fig. 7 ICS II inhibited cerebral I/R-induced neural apoptosis in rats. a Representative images of TUNEL staining in the hippocampus and cortex. b Quantitative analysis of TUNEL-positive cells in the hippocampus and cortex. c Representative images of immunoblotting of Bax and $\mathrm{Bcl}$-2. d Representative images of immunoblotting of caspase 3 and cleaved-caspase 3 in the penumbra. e Quantitative analysis of Bax/Bcl-2. f Quantitative analysis of cleaved-caspase 3/caspase 3. Three to five random microscope fields per section were chosen. The average was calculated. ${ }^{* *} P<0.01$ vs sham; ${ }^{\# \#} P<0.01$ vs MCAO; $n=6$ per group.

\section{ICS II bound to and inhibited MMP2/9}

Molecular docking analysis was applied to confirm whether ICS II binds to the MMP2 and MMP9 proteins. Our findings demonstrated that the binding energy of ICS II and MMP2 and of ICS II and MMP9 were $-6.89 \mathrm{kcal} / \mathrm{mol}$ and $-10.25 \mathrm{kcal} / \mathrm{mol}$, respectively, which verified that ICS II directly bound to MMP2 and MMP9 (Fig. 8). We subsequently further explored the possible binding modes and interactions within the amino acid pocket, including Glu A57, Met A61, Asp A71, Gly A91, Arg A18, His A97 and Asp A95 of MMP2 and Met A247, Tyr A218, His A236, Leu A188, Pro B246, Val A223, Gln A227 and Gln B227 of MMP9 (Fig. 8). These results indicated that ICS II might directly affect MMP2 and MMP9 to attenuate BBB dysfunction.

\section{DISCUSSION}

The present study demonstrated that (1) ICS II significantly reduced $B B B$ disruption after cerebral I/R; (2) ICS II not only reduced the expression and activity of MMP2/9 but also increased TIMP1 and tight junction protein expression; and (3) the inhibitory effect of ICS II on BBB disruption after cerebral I/R was, at least partly, mediated by a caspase 3-dependent pathway (Fig. 9).

Cerebral ischemia and subsequent reperfusion result in harmful consequences in ischemic stroke, especially disruption of the BBB, which leads to severe hemorrhagic transformation and poor prognosis through aggravation of edema formation and brain hemorrhage [24-26]. BBB dysfunction also increases mortality following tPA treatment after stroke, especially following delayed tPA treatment [27]. Our results suggested that ICS II attenuates BBB dysfunction, as evidenced by Evans Blue staining. However, the detailed mechanisms need to be explored in depth.

MMPs, also called matrixins, are enzymes characterized by a zinc ion binding motif in the catalytic domain and several conserved protein domains that play a crucial role in early vascular remodeling and destabilization of atherosclerotic plaques in brain arteries [28-30]. TIMPs, physiological and specific tissue inhibitors of MMPs, tightly regulate the activation and function of MMPs [31, 32].
Under physiological conditions, there is a balanced interaction between MMPs and TIMPs, which is essential for development and extracellular matrix homeostasis [33]. TIMP1, an endogenous inhibitor of MMP9, inhibits MMP9 activity via high-affinity and noncovalent binding to the MMP catalytic domain [34, 35]. However, plenty of evidence suggests that MMP9 and TIMP1 are associated with brain infarction or edema and dysfunction of the BBB after ischemic stroke [36-38]. Moreover, intravenous treatment with tPA can also produce catastrophic hemorrhagic transformation in the ischemic brain by triggering MMP9 activation [39]. Consistent with our previous studies, the results indicated that $\mathrm{I} / \mathrm{R}$ induced increases in MMP2/9 activities and protein expression and decreased TIMP1 protein expression in MCAO rats $[40,41]$. However, ICS II significantly inhibited this change. Since the striatum is the first region of the brain affected by ischemia and reperfusion in the MCAO model followed by the cortex [42], the localization of MMP2/9 and TIMP1 in the striatum and cortex was determined using immunohistochemical staining. As expected, MMP2/9 were significantly increased and TIMP1 was decreased in both the striatum and cortex after MCAO insult. Furthermore, the results also showed that ICS II directly bound to $\mathrm{MMP} 2 / 9$, as evidenced by molecular docking, which further indicated that MMP2/9 plays an imperative role in the beneficial effect of ICS II on cerebral I/R-induced injury, especially disruption of the BBB.

In addition, tight junctions perform dual fence and barrier functions and are the structural basis of the BBB [43, 44]. Tight junction proteins, such as claudin 5, occludin and ZO 1, are critical determinants of the permeability properties of the BBB [45]. Multiple lines of evidence suggest that tight junctions are related to disruption of the BBB [46-48]. Our results showed that cerebral I/R injury decreased the expression of claudin 5, occludin, and ZO 1 , which is consistent with a previous report [49]. However, ICS II significantly reversed the change, which suggests that ICS II upregulates tight junction proteins, therefore protecting the integrity of the BBB.

Of note, cerebral I/R is known to be accompanied by apoptosis, and TIMP1 could prevent cell apoptosis $[50,51]$. Hence, neural 

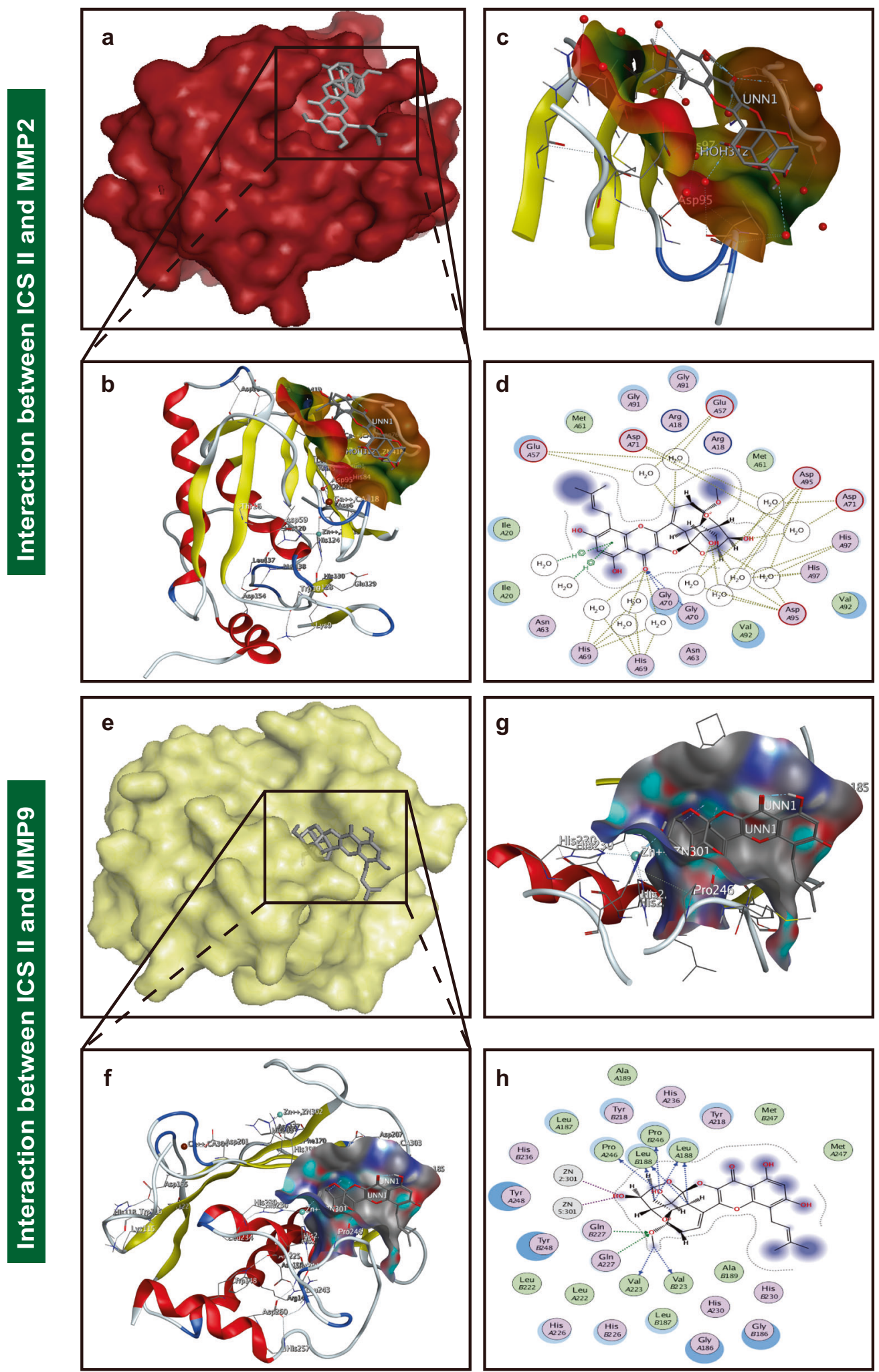

Fig. 8 ICS II bound and inhibited MMP2/9. a A visual of the binding sites between ICS II and MMP2. b A close-up of the molecule binding pocket from the side. c The crystal structure of ICS II (gray) displaying MMP2 (yellow and purple) bound to the docking pocket. d Amino acid residues. e A visual of the binding sites between ICS II and MMP9. f A close-up of the molecule binding pocket from the side. $\mathbf{g}$ The crystal structure of ICS II (gray) displaying MMP9 (yellow and purple) bound to the docking pocket. $\mathbf{h}$ Amino acid residues. 


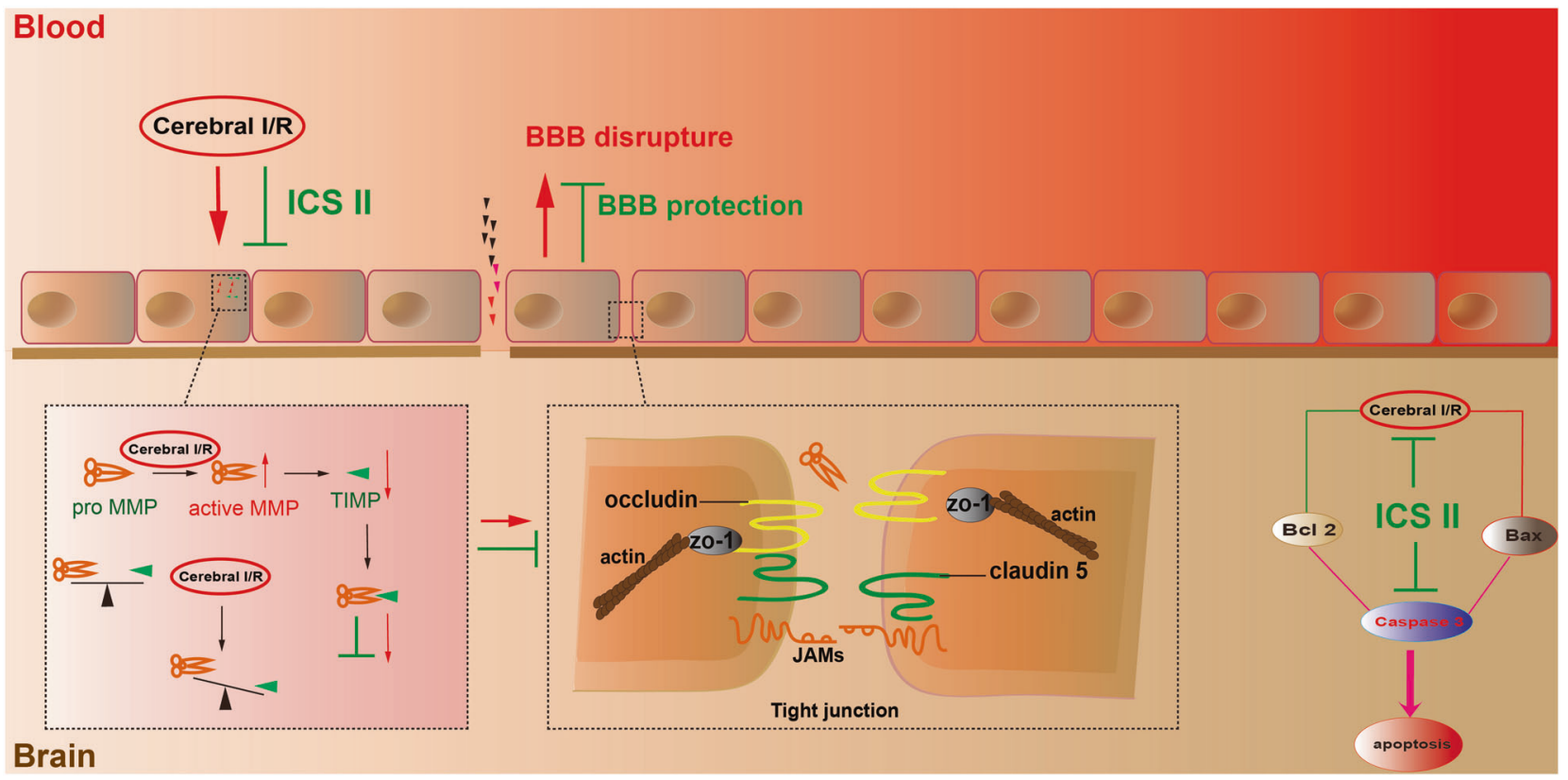

Fig. 9 A schematic showing the underlying mechanisms of the inhibitory effects of ICS II on cerebral I/R-induced apoptosis in rats. ICS II attenuates cerebral I/R-induced BBB dysfunction by inhibiting neuronal apoptosis in rats in a manner involving regulation of the MMP9/TIMP1 balance.

apoptosis in the hippocampus and cortex was examined. The results demonstrated that the number of apoptotic cells was significantly increased after MCAO, as evidenced by the TUNEL assay. However, ICS II obviously reduced the number of apoptotic cells. Furthermore, ICS II not only increased the ratio of Bax/Bcl-2 but also decreased the level of active caspase 3 . These findings suggest that ICS II significantly suppresses cerebral I/R-induced neural apoptosis.

In summary, the current study revealed that ICS II significantly ameliorates $\mathrm{I} / \mathrm{R}$-induced $\mathrm{BBB}$ disruption in $\mathrm{MCAO}$ rats by regulating the balance of MMP9/TIMP1 and further inhibiting neuronal apoptosis by a caspase 3-dependent apoptosis pathway, thereby protecting against cerebral I/R injury.

\section{ACKNOWLEDGEMENTS}

This work was supported by the National Natural Science Foundation of China (grant No. 81760727), the National Key R\&D Plan for Research on Modernization of Traditional Chinese Medicine (grant No. 2017YFC1702005), the Post Subsidy Project of State Key R\&D Plan in the Social Development Field (grant No. SQ2017YFC17020405), the "Hundred" Level Of High-level Innovative Talents in Guizhou Province (grant No. QKHRCPT 20165684), the Program for Changjiang Scholars and Innovative Research Team in University, China (grant No. IRT_17R113), and the Program for Outstanding Youth of Zunyi Medical University (grant No. 15zy-002).

\section{AUTHOR CONTRIBUTIONS}

QHG and JSS designed the experimental protocols. MBL and WW carried out all the studies. MBL wrote the paper with help from QHG, FL and JMG.

\section{ADDITIONAL INFORMATION}

Competing interests: The authors declare no competing interests.

\section{REFERENCES}

1. Zhang QY, Wang ZJ, Sun DM, Wang Y, Xu P, Wu WJ, et al. Novel therapeutic effects of leonurine on ischemic stroke: new mechanisms of BBB integrity. Oxid Med Cell Longev. 2017;2017:7150376.
2. Bayraktutan U. Endothelial progenitor cells: potential novel therapeutics for ischaemic stroke. Pharmacol Res. 2019;144:181-91.

3. Muir KW. Stroke in 2015: the year of endovascular treatment. Lancet Neurol 2016;15:2-3.

4. Chapman SN, Mehndiratta P, Johansen MC, McMurry TL, Johnston KC, Southerland AM. Current perspectives on the use of intravenous recombinant tissue plasminogen activator (tPA) for treatment of acute ischemic stroke. Vasc Health Risk Manag. 2014;10:75-87.

5. Mao L, Li P, Zhu W, Cai W, Liu Z, Wang Y, et al. Regulatory T cells ameliorate tissue plasminogen activator-induced brain haemorrhage after stroke. Brain. 2017;140:1914-31.

6. Karatas H, Eun Jung J, Lo EH, van Leyen K. Inhibiting 12/15-lipoxygenase to treat acute stroke in permanent and TPA induced thrombolysis models. Brain Res. 2018;1678:123-8.

7. Murata Y, Rosell A, Scannevin RH, Rhodes KJ, Wang X, Lo EH. Extension of the thrombolytic time window with minocycline in experimental stroke. Stroke. 2008;39:3372-7.

8. Zhang L, Chopp M, Jia L, Cui Y, Lu M, Zhang ZG. Atorvastatin extends the therapeutic window for tPA to $6 \mathrm{~h}$ after the onset of embolic stroke in rats. J Cereb Blood Flow Metab. 2009;29:1816-24.

9. Wang W, Li M, Chen Q, Wang J. Hemorrhagic transformation after tissue plasminogen activator reperfusion therapy for ischemic stroke: mechanisms, models, and biomarkers. Mol Neurobiol. 2015;52:1572-9.

10. Peluffo $H$, Unzueta U, Negro-Demontel ML, Xu Z, Vaquez E, Ferrer-Miralles $N$, et al. BBB-targeting, protein-based nanomedicines for drug and nucleic acid delivery to the CNS. Biotechnol Adv. 2015;33:277-87.

11. Campos-Bedolla P, Walter FR, Veszelka S, Deli MA. Role of the blood-brain barrier in the nutrition of the central nervous system. Arch Med Res. 2014;45:610-38.

12. Jeffrey $P$, Summerfield $S$. Assessment of the blood-brain barrier in CNS drug discovery. Neurobiol Dis. 2010;37:33-7.

13. Kong LL, Wang ZY, Hu JF, Yuan YH, Li H, Chen NH. Inhibition of chemokine-like factor 1 improves blood-brain barrier dysfunction in rats following focal cerebral ischemia. Neurosci Lett. 2016;627:192-8.

14. Jiang $X$, Andjelkovic AV, Zhu L, Yang T, Bennett MVL, Chen J, et al. Blood-brain barrier dysfunction and recovery after ischemic stroke. Prog Neurobiol. 2018;163164:144-71.

15. Jin R, Yang G, Li G. Molecular insights and therapeutic targets for blood-brain barrier disruption in ischemic stroke: critical role of matrix metalloproteinases and tissue-type plasminogen activator. Neurobiol Dis. 2010;38:376-85.

16. Sandoval KE, Witt KA. Blood-brain barrier tight junction permeability and ischemic stroke. Neurobiol Dis. 2008;32:200-19. 
17. Li C, Li Q, Mei Q, Lu T. Pharmacological effects and pharmacokinetic properties of icariin, the major bioactive component in Herba Epimedii. Life Sci. 2015;126:57-68.

18. Zhang D, Zhang J, Fong C, Yao X, Yang M. Herba Epimedii flavonoids suppress osteoclastic differentiation and bone resorption by inducing G2/M arrest and apoptosis. Biochimie. 2012;94:2514-22.

19. Liu YQ, Yang QX, Cheng MC, Xiao HB. Synergistic inhibitory effect of icariside II with icaritin from Herba Epimedii on pre-osteoclastic RAW264.7 cell growth. Phytomedicine. 2014;21:1633-7.

20. Liu S, Li X, Gao J, Liu Y, Shi J, Gong Q. Icariside II, a phosphodiesterase-5 inhibitor, attenuates beta-amyloid-induced cognitive deficits via BDNF/TrkB/CREB signaling. Cell Physiol Biochem. 2018;49:985.

21. Zhou J, Deng Y, Li F, Yin C, Shi J, Gong Q. Icariside II attenuates lipopolysaccharide-induced neuroinflammation through inhibiting TLR4/MyD88/ NF-kappaB pathway in rats. Biomed Pharmacother. 2019;111:315-24.

22. Gao J, Long L, Xu F, Feng L, Liu Y, Shi J, et al. Icariside II, a phosphodiesterase 5 inhibitor, attenuates cerebral ischemia/reperfusion injury by inhibiting GSK-3 $\beta$ mediated activation of autophagy. Br J Pharmacol. 2020;177:1434-52.

23. Deng Y, Xiong D, Yin C, Liu B, Shi J, Gong Q. Icariside II protects against cerebral ischemia reperfusion injury in rats via nuclear factor-kB inhibition and peroxisome proliferator activated receptor up-regulation. Neurochem Int. 2016;96:56-61.

24. Borlongan CV, Rodrigues AA Jr, Oliveir MC. Breaking the barrier in stroke: what should we know? A mini-review. Curr Pharmacol Des. 2012;18:3615-23.

25. Kastrup A, Groschel K, Ringer TM, Redecker C, Cordesmeyer R, Witte O, et al. Early disruption of the blood-brain barrier after thrombolytic therapy predicts hemorrhage in patients with acute stroke. Stroke. 2008;39:2385-7.

26. Potrovita I, Zhang W, Burkly L, Hahm K, Lincecum J, Wang M, et al. Tumor necrosis factor-like weak inducer of apoptosis-induced neurodegeneration. J Neurosci. 2004;24:8237-44.

27. Jickling GC, Liu D, Stamova B, Ander B, Zhan X, Lu A, et al. Hemorrhagic transformation after ischemic stroke in animals and humans. J Cereb Blood Flow Metab. 2014;34:185-99.

28. Gaubatz JW, Ballantyne CM, Wasserman BA, He M, Chambless LE, Boerwinkle E, et al. Association of circulating matrix metalloproteinases with carotid artery characteristics: the atherosclerosis risk in communities carotid MRI study. Arterioscler Thromb Vasc Biol. 2010;30:1034-42.

29. Hannocks MJ, Zhang X, Gerwien H, Chashchina A, Burmeister M, Korpos E, et al. The gelatinases, MMP-2 and MMP-9, as fine tuners of neuroinflammatory processes. Matrix Biol. 2019;75-76:102-13.

30. Nagase $H$, Visse $R$, Murphy $G$. Structure and function of matrix metalloproteinases and TIMPs. Cardiovasc Res. 2006;69:562-73.

31. Jäsberg H, Tervahartiala T, Sorsa T, Söderling E, Haukioja A. Probiotic intervention influences the salivary levels of matrix metalloproteinase (MMP)-9 and tissue inhibitor of metalloproteinases (TIMP)-1 in healthy adults. Arch Oral Biol. 2018;85:58-63.

32. Bourboulia D, Stetler-Stevenson WG. Matrix metalloproteinases (MMPs) and tissue inhibitors of metalloproteinases (TIMPs): positive and negative regulators in tumor cell adhesion. Semin Cancer Biol. 2010;20:161-8.

33. Zielinska-Turek J, Dorobek M, Turek G, Barcikowska-Kotowicz M. MMP-9 and/or TIMP as predictors of ischaemic stroke in patients with symptomatic and asymptomatic atherosclerotic stenosis of carotid artery treated by stenting or endarterectomy - a review. Neurol Neurochir Pol. 2018;52:555-61.
34. Brew K, Dinakarpandian D, Nagase H. Tissue inhibitors of metalloproteinases: evolution, structure and function. Biochim Biophys Acta. 2000;1477:267-83.

35. Nosrati R, Kheirouri S, Ghodsi R, Ojaghi H. The effects of zinc treatment on matrix metalloproteinases: a systematic review. J Trace Elem Med Biol. 2019;56:107-15.

36. Fujimoto $M$, Takagi $Y$, Aoki $T$, Hayase $M$, Marumo T, Gomi M, et al. Tissue inhibitor of metalloproteinases protect blood-brain barrier disruption in focal cerebral ischemia. J Cereb Blood Flow Metab. 2008;28:1674-85.

37. Wei H, Wang S, Zhen L, Yang Q, Wu Z, Lei X, et al. Resveratrol attenuates the blood-brain barrier dysfunction by regulation of the MMP-9/TIMP-1 balance after cerebral ischemia reperfusion in rats. J Mol Neurosci. 2015;55:872-9.

38. Liu Y, Wang D, Wang H, Qu Y, Xiao X, Zhu Y. The protective effect of HET0016 on brain edema and blood-brain barrier dysfunction after cerebral ischemia/reperfusion. Brain Res. 2014;1544:45-53.

39. Adibhatla RM, Hatcher JF. Tissue plasminogen activator (tPA) and matrix metalloproteinases in the pathogenesis of stroke: therapeutic strategies. CNS Neurol Disord Drug Targets. 2008;7:243-53.

40. Fu S, Gu Y, Jiang JQ, Chen X, Xu M, Chen X, et al. Calycosin-7-O- $\beta$ - $D$-glucoside regulates nitric oxide/caveolin-1/matrix metalloproteinases pathway and protects blood-brain barrier integrity in experimental cerebral ischemia-reperfusion injury. J Ethnopharmacol. 2014;155:692-701.

41. Li XF, Zhang XJ, Zhang C, Wang LN, Li Y, Zhang Y, et al. Ulinastatin protects brain against cerebral ischemia/reperfusion injury through inhibiting MMP-9 and alleviating loss of ZO-1 and occludin proteins in mice. Exp Neurol. 2018;302:68-74.

42. Traystman RJ. Animal models of focal and global cerebral ischemia. ILAR J. 2003;44:85-95.

43. Zhang YM, Zhou Y, Qiu LB, Ding GR, Pang XF. Altered expression of matrix metalloproteinases and tight junction proteins in rats following PEMF-induced BBB permeability change. Biomed Environ Sci. 2012;25:197-02.

44. Li C, Wang X, Cheng F, Du X, Yan J, Zhai C, et al. Geniposide protects against hypoxia/reperfusion-induced blood-brain barrier impairment by increasing tight junction protein expression and decreasing inflammation, oxidative stress, and apoptosis in an in vitro system. Eur J Pharmacol. 2019;854:224-31.

45. Tietz S, Engelhardt B. Brain barriers: crosstalk between complex tight junctions and adherens junctions. J Cell Biol. 2015;209:493-506.

46. Lv J, Hu W, Yang Z, Li T, Jiang S, Ma Z, et al. Focusing on claudin-5: a promising candidate in the regulation of BBB to treat ischemic stroke. Prog Neurobiol. 2018;161:79-96.

47. Haseloff RF, Dithmer S, Winkler L, Wolburg H, Blasig IE. Transmembrane proteins of the tight junctions at the blood-brain barrier: structural and functional aspects. Semin Cell Dev Biol. 2015;38:16-25.

48. Huber JD, Egleton RD, Davis TP. Molecular physiology and pathophysiology of tight junctions in the blood-brain barrier. Trends Neurosci. 2001;24:719-25.

49. Li Y, Zhong W, Jiang Z, Tang X. New progress in the approaches for blood-brain barrier protection in acute ischemic stroke. Brain Res Bull. 2019;144:46-57.

50. El Khashab IH, Abdelsalam RM, Elbrairy Al, Attia AS. Chrysin attenuates global cerebral ischemic reperfusion injury via suppression of oxidative stress, inflammation and apoptosis. Biomed Pharmacother. 2019;112:108619.

51. Stetler-Stevenson WG. Tissue inhibitors of metalloproteinases in cell signaling: metalloproteinase-independent biological activities. Sci Signal 2008;1:re6. 